\title{
The Effects of Typing Demand on Learner's Motivation/Attitude-Driven Behaviour (MADB) Model with Mouse and Keystroke Behaviours
}

\author{
Yee Mei Lim \\ Faculty of Computing and Information Technology \\ Tunku Abdul Rahman University College \\ Kuala Lumpur, Malaysia \\ Email:ymlim@acd.tarc.edu.my
}

\author{
Aladdin Ayesh \\ Faculty of Technology \\ De Montfort University \\ Leicester, United Kingdom \\ Email: aayesh@dmu.ac.uk
}

\author{
Martin Stacey \\ Faculty of Technology \\ De Montfort University \\ Leicester, United Kingdom \\ Email: mstacey@dmu.ac.uk
}

\begin{abstract}
It would be desirable to have an automated means of assessing a learner's motivation and stress levels in an elearning system, which would give impact on his or her learning performance. This preliminary research examines the effects of typing task demand on Motivation/Attitude-driven Behavior (MADB) model. The model is adapted from what was proposed by Wang [1], which is used to describe how the motivation process drives human behaviours and actions, and how the attitude and decision-making process help to regulate and determine the action to be taken by the learner. The effects of typing demand are tested on learners' stress perceptions, motivation, attitudes, decision, as well as their mouse and keystroke behaviours. The typing demand is varied by the pre-defined text length and language familiarity. The results of Multivariate Analysis of Variance and correlation tests are generally congruent with the MADB model proposed by Wang, but with minor difference. We also found that a learner's behaviour is significantly correlated to his or her mouse and keystroke behaviours. A revised version of MADB model based on e-learning environment is proposed.
\end{abstract}

Keywords -attitude; behaviour; cognitive processes of the brain; job familiarity; keystroke behaviour; mouse behaviour; motivation; typing

\section{INTRODUCTION}

Affective computing, as part of human-computer interaction research in learning, takes into account a user's emotional states in order to increase learners' motivation, which could transform education [2], [3]. Existing e-learning systems solely rely on learners' scores and time spent on a task to assess a learner's performance. This is not enough to help teachers to identify how a learning content or a task demand affects their learners' engagement and emotion. Unpleasant or negative emotion is believed inhibiting necessary resources to be recruited for further cognitive process by human mental, which prevent optimal skill execution [4]. Hajcak et al [5] argue that negative emotion may be caused by the task demands itself, or by other external factors that are related to the task. If the factor that generates negative emotion can be determined, e-learning developers can redesign the learning process, including adapting the instructions and improving learning environment, to enhance student's attitude in learning. Therefore it is important to study how emotion can be affected by certain factors, such as task demand and external psychophysiological stimuli, how it affects learning performance, and how to enable affect to be computed automatically to enable adaptive learning.

To achieve the afore-mentioned, a few challenges must be overcome. First, the existing affective computing approaches, such as physiological measures and audio-visual computing, are either obtrusive, expensive or need special setup, which are not feasible to be implemented as part of a normal online system. A cheap, ubiquitous and less invasive means of estimating users' emotion must be sought. Second, existing affective learning research considers emotion from multi-dimension. It may be important to have better understanding of granularity of emotion of learner [6]. However, enabling measurement of rich granularity of emotion is extremely challenging [7]. Third, numerous existing psychological research reported the effects of stress on job performance and behaviour [8]-[10], but there is a lack of empirical affective learning research that examines the relationships between learner's stress, cognitive behaviour and learning performance, although many other emotions have been studied. It is important to study the effects of task demand and external psycho-physiological stimuli on learner's stress and learning performance, since stress could result in negative feelings of fear, anxiety and frustration, which build psychological barrier to further learning [7]. Therefore this would be interesting and useful if stress can be measured automatically, as stress could be related to both cognitive stress and emotional stress. Fourth, some research over the past decade started to examine the potential of using mouse or keystroke dynamics [11]-[14], but most of them consider these methods in isolation. The unification of both techniques is important as there is a risk of collecting misleading information from only one channel, since not all tasks require the use of a single device. Furthermore, there is only a little research examines the correlations of a learner's emotions to his/her mouse and keystroke dynamics, although most of them found significant impacts of emotions on learners' mouse/keystroke behaviours. However, there is almost no research has been carried out to study the correlations of learner's stress to the learners' behaviours when using these devices to carry out some tasks in an e-learning environment.

\section{RESEARCH HyPOTHESIS}

To examine the effects of typing task demand and external stimuli on the Motivation/Attitude-driven Behavior (MADB) 
model that is adopted from Wang [1], three hypotheses are given as follows:

1) Typing task demand that includes text length and language familiarity, and external stimuli, i.e. time constraint, clock display and countdown timer, have significant effects on learner's stress perception and motivation.

2) The correlations between typing task demand, external stimuli, stress perception, motivation, rational motivation, attitude, decision, and behaviour are significant.

3) Behaviour significantly affects mouse behaviour $B(M)$ and keystroke behaviour $B(K)$.

A case study is done with the assistance of 162 students from a higher education institution in Malaysia.

\section{MEASURING LEARNER'S Motivation/ATTITUDE-DRIVEN BEHAVIOUR IN LEARNING}

The Motivation/Attitude-driven Behavior (MADB) model was applied in software engineering organization by Wang [1]. According to Wang, motivation can be weaken by unpleasant experience with the system, or poor job performance (outcome). Attitude includes user's confidence with the task based on experience, the estimated effort to complete the task, or the amount of attention can be spent on a task. The combination of motivation and attitude gives impact on the rational motivation, which enables a person to continue doing the task if it is still within their acceptable effort to invest. Decision is affected by time, resources and energy. Time constraint and projected long completion time may reduce their estimated probability of success. The combination of rational motivation and decision will affect the behaviour and task outcome. The task outcome affects the student's motivation and stress perception for carrying out next task.

The previous studies by Lim et al [15]-[17] studied the effects of different tasks on learners' job performance and mouse/keystroke dynamics in an e-learning environment, i.e. searching for a learning material, assessment using mental arithmetic, and typing fixed text with varied length and familiarity. The first case study based on search task was carried out with 151 undergraduate students. The second and third experiments based on mental arithmetic and typing tasks were conducted with 60 students respectively. Based on the three case studies, the following are concluded in general.

1) If task demand increased, then the user stress perception, duration spent for a question, error rate, passive attempt, mouse idle duration may increase, but mouse speed, left mouse click and keystroke speed would decrease generally.

2) The correlation between job performance and mouse behaviour is significant. Low job performance, for instance when the students attempt to revisit the task, give up, or when they make more errors, it usually comes together with longer mouse idle duration, and higher mouse speed. When the student has to perform the task with longer duration, then longer mouse idle duration and slower mouse speed could be observed.

3) Significant correlations between stress perception and mouse/keystroke dynamics are found in all tasks.

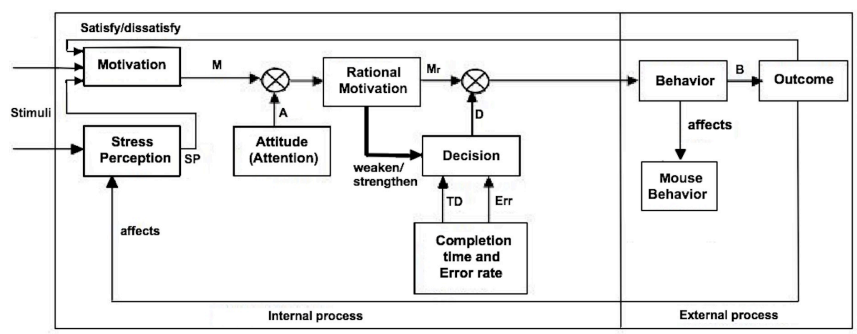

Fig. 1. The Revised MADB Model in the E-Learning Context with Mouse Behaviours

4) The estimation of the emotional stress level based on job performance, mouse dynamics and keystroke dynamics might only be valid as long as the students are still engaged with the task. Once a student's stress level has gone beyond limit, or he or she has lost motivation, anomalous behaviours could be observed.

5) Task demand is the main factor that affects job performance, stress perception, mouse behaviour and keystroke behaviour.

Based on the above findings, Lim et al [18] explored the application of MADB model adapted to an e-learning environment, with added mouse behaviour. They assumed that indirect task demand, such as searching for the correct course module, and external stimuli, such as menu design, have effects on learners' motivation and stress perception, as well as their mouse behaviour. Their findings were generally congruent with what was proposed by Wang. They then revised the MADB model suited for e-learning environment, which is shown in Figure 1.

\section{Methodology}

This research continues the previous work by Lim et al [18], to study the effects of typing demand on learners' MADB model, as well as their mouse and keystroke behaviours. Accordingly, experiments are set to explore how formal cognitive processes are affected by the typing task and three external stimuli in an e-learning system. The demand of the typing task is elevated by increasing the length of the pre-defined texts for the participants to type. In order to simulate the familiar task and unfamiliar task effects, English is introduced as a language that the learners are familiar with, and German language that they are totally unfamiliar with. There are a total of 6 questions (Demand) with various text length (Length) and language familiarity (Familiarity) to be typed in the typing task. The detailed setting of the typing task demand is presented in Table I. Three external stimuli are invoked by imposing time constraint (Timing), and/or display of a clock (Clock), and/or a countdown timer that flashes every second (Timer). Cognitive states are measured based on the Motivation/Attitude-Driven Behaviour (MADB) model adapted from what was proposed by Wang [1]. Learners' stress perceptions on the tasks are gathered using a user self-report with 7-Likert scale. The participants are assigned to 5 different groups randomly, i.e. Group 000, Group 100, Group 101, Group 110 and Group 111. Group 000 is not given any time constraint, and the rest are given 30 seconds for each question. Group 101 has a countdown timer display, Group 110 has a clock display, while Group 111 has both displayed on the screen. 


\section{A. Employment of MADB Model in the Typing Task}

The revised Motivation/Attitude-driven Behaviour (MADB) model by Lim [18] was adopted in this research, with a slight modification with added keystroke behaviour. The proposed MADB model determines the following. First, direct instruction and external stimuli can affect a learner's emotion, i.e. stress. For the direct instruction design, we leverage typing task with different lengths and languages to induce emotional stress. The task demand is increased from Question 1 to Question 6 according to the increment of text length, and familiarity of a language (see Table I). We set up a 30-second time constraint, a digital clock display and a countdown timer that flashes every second in yellow background as the external stress stimuli to the participants.

TABLE I. TYPING TASK DEMAND

\begin{tabular}{|l|c|c|c|c|c|}
\hline \multirow{2}{*}{ Q. } & \multirow{2}{*}{ Language } & \multicolumn{2}{|c|}{ Characteristics } & \multicolumn{2}{c|}{ Length } \\
\cline { 3 - 6 } & length & familiarity & word & letters \\
\hline 1 & English & short & familiar & 5 & 21 \\
\hline 2 & German & short & unfamiliar & 5 & 25 \\
\hline 3 & English & medium & familiar & 20 & 94 \\
\hline 4 & German & medium & unfamiliar & 20 & 99 \\
\hline 5 & English & long & familiar & 63 & 459 \\
\hline 6 & German & long & unfamiliar & 63 & 451 \\
\hline
\end{tabular}

We then measure the strength of stress, i.e. stress perception $S P$, using 7-Likert scale (1 indicates strongly disagree that he/she is stressed, and 7 indicates strongly agree).

$$
1 \leq S P \leq 7
$$

The strength of a motivation, $M$, is proportional to both the strength of stress $S P$ and the desire to continue the task $E$ ( $E=1$ if the learner gives up the current task, else $E=0)$, and the current status $S$ of a person $(S=$ total number of attempts that a person gave up the previous tasks).

$$
M=100-\frac{S P+E+S}{C}
$$

where $C=$ the cost to accomplish the expected motivation, which is averaged by the number of tasks given. $C$ is included to normalize the value of $M$ in the scope of [0..100]. For instance, if the maximum value of $S P=7$, maximum $E=1$ and maximum $S=5$, then $C=17 / 100=0.17$. Lower value of $M$ indicates low motivation, and higher $M$ means stronger motivation.

The mode of an attitude, $A$, as the amount of attention to be spent on a task (in the scope of [0..5]). $A$ is low if the learner attempts to wait until the time is up instead of submitting the answer earlier (passive attempt $=1$ if true, else 0 ). $A$ is measured as follows:

$$
A=5-\text { passive attempt }
$$

A rational motivation $M r$ (in the scope of [0..1]) is defined as a motivation regulated by an attitude $A$ (with a positive or negative judgment).

$$
M r=\frac{M \times A}{500}
$$

For instance, if $M=100$ and $A=5$, then $M r=1$.
A decision $D$ is affected by the availability of time or errors made. If the task duration $T D$ or the accumulated error rate $E r r$ is high, then $D$ would be lower. $E r r$ is the accumulated average typo mistakes, which is scaled with the $\log _{10}$ function.

$$
D=1-\max (T D, E r r)
$$

Lastly, behaviour $B$ is driven by a rational motivation $\mathrm{Mr}$ and supported by a positive decision $D$ toward the action. Based on the MADB model, the outcome of $B$ determines the motivation to continue the task. We also assume that $B$ relatively changes mouse and keystroke behaviours.

$$
B=\min (M r, D)
$$

\section{B. Formulation of Mouse Behaviour and Keystroke Behaviour}

We define the mouse behaviour as a dataset that captures the mouse features for each task. The mouse behaviour is defined as follows:

$$
B(M)=<M S, M I D, M I O, M C>
$$

$M S=$ Average mouse speed (pixels per ms)

$M I D=$ Total mouse inactivity duration $(\mathrm{ms})$

$M I O=$ Total mouse inactivity occurrences

$M C=<M C L, M C R^{1}>$, which is a dataset that consists of left mouse press rate $(M C L)$ and right mouse press rate $(M C R)$.

Lastly we define the keyboard behaviour as a dataset that captures the keyboard features for each task, as follows:

$$
B(K)=<K L, K S, K E r r>
$$

$K L=$ Average key latency (ms)

$K S=$ Average typing speed per key (per second)

$K E r r=K B S+K D$, the total occurrences of error keys used, which includes backspace $(K B S)$ and delete $(K D)$ keys

\section{Procedures}

We set up the experiments in a computer laboratory of an institution in Malaysia, which was equipped with 24 computers that run on Windows 7, with 17" LCD monitor (resolution of $1024 \times 768$ pixels). All computers were using a standard external HID-compliant mouse. The Web pages run on Google Chrome by default. Before the participants start the actual typing task, a general instruction is displayed to inform what they should do in the task. During the typing task, the participants are required to type the pre-defined text into a textbox. There are 6 questions with various text lengths, which 3 questions in English and 3 in German, as explained in Table I. To force the use of mouse so that mouse dynamics could be collected, the Enter key is disabled so that the participants must use a mouse to click on the Save button to submit the page. For the experimental groups who are given a time constraint, if they do not click the Save button before the time is up, the page will be submitted automatically when the time limit is reached. If the page is submitted automatically by the system, then attention $A$ (as defined in Equation 3) will be computed. Anyone who wishes to skip to the next question, they may click the Give Up button on the top right corner ( $E$ is collected as in Equation 2 ). The amount of typo mistakes made by a participant in a

\footnotetext{
${ }^{1} M C R$ was removed later due to no data.
} 
given text upon submission is counted and scaled using the $\log _{10}$ function. The accumulated average error rate Err based on the scaled typo mistakes is then computed, as in Equation 5. Upon completion of every question, or when the Give Up button is pressed, the end time (in millisecond) is recorded, and task duration $T D$ is computed, which is used in Equation 5. Then the next question will be displayed according to the predetermined order as shown in Table I. Every time a participant completed a question, a self-report survey is displayed as follows:

\author{
"You felt stressed when answering the previous \\ question"
}

The participant must indicate his or her perception of the stress level $S P$ when solving the problem, following the 7-point Likert scale ( 1 for strongly disagree, 7 for strongly agree), as defined in Equation 1.

\section{Samples}

As this research focuses on e-learning environment, we draw participants from the likely users of the actual learning management system. We conducted the experiments within 2 weeks with 14 different groups of students. Each session of the experiments took about 5 minutes. Hundred and ninety students from Bachelor Degree in Computer Science, Bachelor Degree in Information Systems, and Bachelor Degree in Information Technology are recruited based on voluntarily basis without any incentive. Only 162 of them completed the typing task. Among these 162 participants, majority are male $(89.51 \%)$, aged $20-29$ years old $(94.44 \%)$, have more than 2 years of experience in the Blackboard e-learning system $(85.80 \%)$, and about $40 \%$ of them use the system for more than 10 times in one term $(40.74 \%)$. There are 32 of them from Group 000, 32 from Group 100 and 101 respectively, 36 from Group 110, and 30 from Group 111. All of them passed the English test in Malaysian Certificate of Education, but none of them know German language. Based on the 162 participants who completed the typing tasks, we achieved 972 sample data $(\mathrm{N}=972)$ for the subsequent statistical analyses.

\section{RESUlT}

The sections below discuss the results of the 3 hypotheses stated in Section II.

\section{A. The Effects of Typing Demand and External Stimuli on User's Stress Perception (SP) and Motivation (M)}

Based on the Univariate Analysis of Variance (ANOVA) test as shown in Table II, the effects of Question, Length, Familiarity, and Timer are significant. There are no significant effects of time constraint and clock display on stress perception $S P$ and motivation $M$ at all. The interactions between effects are insignificant. $S P$ increased and $M$ decreased significantly when the task demand is elevated from Question 1 to Question 6. $S P$ increased and $M$ reduced significantly when text length increased. When familiar language is introduced, $S P$ reduced and $M$ increased significantly. In terms of external stimuli, for those who are given a countdown timer, their $S P$ is generally higher and $M$ is lower than others.
TABle II. Test Between Question, Timing, Clock and Timer SIGNIFICANT EFFECTS ON $S P$ AND $M$

\begin{tabular}{|l|l|l|c|c|c|}
\hline Factor & $\begin{array}{l}\text { Sample } \\
\text { Size }\end{array}$ & $\boldsymbol{N}$ & $\boldsymbol{p}(\mathbf{S P})$ & $\boldsymbol{p}(\boldsymbol{M})$ \\
\hline $\begin{array}{l}\text { Typing } \\
\text { Demand }\end{array}$ & Question & All & 972 & $\mathbf{. 0 3} e^{-37}$ & $\mathbf{. 0 8} e^{-39}$ \\
\cline { 2 - 6 } & Length & All & 972 & $\mathbf{. 0 7} e^{-37}$ & $\mathbf{. 0 2} e^{-38}$ \\
\cline { 2 - 6 } & Familiarity & All & 972 & $\mathbf{. 0 0 1 2}$ & $\mathbf{. 0 0 0 8}$ \\
\hline \multirow{3}{*}{$\begin{array}{l}\text { External } \\
\text { Stimuli }\end{array}$} & Timing & 000,100 & 384 & .6282 & .9831 \\
\cline { 2 - 6 } & Clock & Timing $=1$ & 780 & .2352 & .3436 \\
\cline { 2 - 6 } & Timer & Timing $=1$ & 780 & $\mathbf{. 0 0 8 4}$ & $\mathbf{. 0 0 7 0}$ \\
\hline \multicolumn{2}{|l|}{ Correlation is significant at $p<.05$ (2-tailed) level (highlighted in bold). All interactions between factors }
\end{tabular}

are not significan

\section{B. The Correlations between Typing Demand, External Stim-} uli, and Cognitive States

The Spearman Correlation test is performed to determine the correlations between typing task demand, external stimuli, i.e. Timing, Clock and Timer, stress perception $S P$ and motivation $M$. The significant correlations between the stress stimuli, $S P$ and $M$ are found. Table III shows that when task demand (Question) or text length (Length) increased, or language familiarity (Familiarity) reduced, then $S P$ increased and $M$ decreased significantly. In terms of external stimuli, only Timer is found correlated to $S P$ and $M$. When timer is displayed, $S P$ increased and $M$ becomes lower significantly. $M$ has an inverse correlation to $S P(p=0)$. When $S P$ increases, $M$ would decrease. $M$ also correlates to attitude $A$. $A$ was computed based on passive attempt in the typing task, i.e. the attempt that a participant would wait until the time is up. The effect of $M$ on $A$ is significant based on a regression test $\left(p=.01 e^{-20}\right)$. Both $M$ and $A$ are correlated to rational motivation $M r . M r$ and decision $D$ are also significantly correlated to behaviour $B$. Both effects of $M r$ and $D$ on $B$ are significant according to regression tests ( $p=0$ and $p=.02 e^{-132}$ respectively). $B$ significantly correlates to $M$ and $S P$. The effects of $B$ on $M$ is also significant from a regression test $\left(p=.03 e^{-293}\right)$. There is also a significant effect of $B$ on $S P\left(p=.09 e^{-291}\right)$, which was also observed during the menu search task reported in [18]. This indicates that $B$ affects both $M$ and $S P$ in both menu search and typing tasks. However, when $B$ improved, lower $S P$ and higher $M$ can be observed in both tasks.

\section{The Effects and Correlations of Behaviour to Mouse Be- haviour and Keystroke Behaviour}

To understand how the changes of behaviour $B$ affects keystroke behaviour $B(K)$ and mouse behaviour $B(M)$, the effects of $B$ on $B(M)$ and $B(K)$ are examined using the Multivariate Analysis of Variance test (MANOVA). The Pearson Correlation test is then conducted to observe the correlations between $B, B(M)$ and $B(K)$. We reduced the sample size and used only Question 1 to Question 4 in the tests, as Question 5 and Question 6 consist of high number of outliers for the mouse and keystroke data. The outliers are caused by the intentional insufficient time constraint given to the participants. Therefore, a sample size of $648(N=648)$ is used in this study.

The MANOVA tests in Table IV show that the effects of Behaviour $B$ on $B(M)$ and $B(K)$ are significant. Wilks' lambda $(\lambda)$ considers differences over all the characteristic roots. The smaller the value of Wilks' lambda, the greater the implied significance [19]. Hence, the effect of $B$ on $B(K)$ is stronger 
than $B(M)$ in the typing task. Since the causation effects of $B$ on $B(M)$ and $B(K)$ are prominent, we study the correlations between $B$ and the features of $B(M)$ and $B(K)$. The result in Table $\mathrm{V}$ shows that $B$ is significantly correlated to $B(M)$ and $B(K)$. When $B$ increased, $M S$ also increased $\left(p=.054 e^{-5}\right)$, $M I O$ increased $\left(p=.07 e^{-20}\right), K S$ increased $(p=.0012)$, but MID, MCL, KL and KErr decreased $\left(p=.06 e^{-19}\right.$, $p=.002$ and $p=.0063$ respectively), which indicate that the student's mouse and keystroke actions become faster when his/her behaviour improves.

TABlE III. CORRElations Between Typing Demand FaCtors AND THE DEPENDENT VARIABLES

\begin{tabular}{|c|c|c|c|c|c|c|}
\hline Factor & SP & $\bar{M}$ & $\mathbf{A}$ & Mr & D & $\bar{B}$ \\
\hline Question & $.05 e^{-40}$ & $.02 e^{-41}$ & $.01 e^{-21}$ & $.03 e^{-50}$ & $.06 e^{-100}$ & $.02 e^{-79}$ \\
\hline Length & $.02 e^{-37}$ & $.01 e^{-38}$ & $.04 e^{-23}$ & $.02 e^{-47}$ & $.07 e^{-96}$ & $.01 e^{-75}$ \\
\hline Familiar & .0012 & .0010 & .0649 & .0008 & $.01 e^{-03}$ & $.01 e^{-3}$ \\
\hline timing & .5914 & .7739 & $.04 e^{-17}$ & .0451 & $.07 e^{-4}$ & .0078 \\
\hline clock & .1638 & .2478 & .7133 & .2928 & .0290 & .4348 \\
\hline timer & .0080 & .0069 & .0941 & .0930 & .3150 & .0059 \\
\hline SP & - & 0 & $.05 e^{-23}$ & 0 & $.01 e^{-36}$ & 0 \\
\hline M & 0 & - & $.01 e^{-21}$ & 0 & $.03 e^{-36}$ & 0 \\
\hline $\mathbf{A}$ & $.05 e^{-23}$ & $.01 e^{-21}$ & - & $.01 e^{-77}$ & $.02 e^{-77}$ & $.04 e^{-}$ \\
\hline $\mathrm{Mr}$ & 0 & 0 & $.01 e^{-77}$ & - & $.05 e^{-61}$ & 0 \\
\hline D & $.01 e^{-36}$ & $.03 e^{-36}$ & $.02 e^{-77}$ & $.05 e^{-61}$ & - & $.08 e^{-}$ \\
\hline B & 0 & 0 & $.04 e^{-78}$ & 0 & $.08 e^{-108}$ & - \\
\hline
\end{tabular}

TABLE IV. The EFFects of Behaviour on Mouse Behaviour AND KEYSTROKE BEHAVIOUR

\begin{tabular}{|c|c|c|c|}
\hline Effect & Dependent Variable & Sig. $p$-value & Wilk's $\lambda$ \\
\hline \multirow[t]{4}{*}{ Mouse Behaviour } & MS & $.03 e^{-06}$ & \multirow[t]{4}{*}{.8221} \\
\hline & MID & $.07 e^{-38}$ & \\
\hline & MIO & $.07 e^{-45}$ & \\
\hline & MCL & $.01 e^{-03}$ & \\
\hline \multirow[t]{3}{*}{ Keystroke Behaviour } & KS & .0056 & \multirow[t]{3}{*}{.3474} \\
\hline & KL & .0416 & \\
\hline & KErr & .0201 & \\
\hline
\end{tabular}

TABle V. Correlations between Behaviour (B), Mouse BEHAVIOUR AND KEYSTROKE BEHAVIOUR

\begin{tabular}{|l|c|c|c|c|c|c|c|c|}
\hline Feature & $\mathrm{B}$ & $\mathrm{MS}$ & MID & MIO & MCL & KS & KL & KErr \\
\hline B & - & $\checkmark$ & $\checkmark$ & $\checkmark$ & $\checkmark$ & $\checkmark$ & $\checkmark$ & $\checkmark$ \\
\hline MS & $.05 e^{-5}$ & - & & & & & & \\
\hline MID & $.06 e^{-19}$ & .077 & - & $\checkmark$ & $\checkmark$ & $\checkmark$ & $\checkmark$ & $\checkmark$ \\
\hline MIO & $.07 e^{-20}$ & .546 & $.06 e^{-29}$ & - & $\checkmark$ & $\checkmark$ & $\checkmark$ & $\checkmark$ \\
\hline MCL & .002 & .633 & .001 & $.02 e^{-03}$ & - & $\checkmark$ & & \\
\hline KS & .0012 & .394 & $.07 e^{-07}$ & .009 & .033 & - & $\checkmark$ & $\checkmark$ \\
\hline KL & .0063 & .860 & $.03 e^{-17}$ & $.066 e^{-03}$ & .546 & $.05 e^{-223}$ & - & \\
\hline KErr & .006 & .190 & $.04 e^{-05}$ & .013 & .750 & .001 & .417 & - \\
\hline
\end{tabular}

Significant correlatio
correlation coefficien

\section{DISCUSSIONS}

Experiments and statistical analyses were conducted to answer the hypotheses, namely (1) typing demand (Question, Length and Familiarity) and external stimuli (Timing, Clock and Timer) have significant effects on stress perception SP and motivation $M$; (2) typing demand and external stimuli are correlated to $S P$ and cognitive states that include motivation $M$, attitude $A$, rational motivation $M r$, decision $D$, and behaviour $B$; and (3) behaviour $B$ are correlated to mouse behaviour $B(M)$ and keystroke behaviour $B(K)$. The results are critically discussed in the following subsections. The outcome of the experiments also validate the consistency between the revised MADB model as proposed in the menu search task in [18], and the typing task in this research.
A. The Effects of Typing Demand and External Stimuli on User's Stress Perception and Cognitive States and Their Correlations

Typing Demand (Question) gives significant impacts on users' stress perception and motivation. As expected, question with longer length and/or low (language) familiarity increase stress perception $S P$ and decrease motivation $M$. Longer text length indicates that the estimated time duration to complete the task would be longer. Humans could be more stressed over time taken to complete a task [15], [20]. The finding of familiarity effects on $S P$ and $M$ also corroborates with the research by Tobias et al [21] and Hulme et al [22]. Tobias et al suggested that lack of familiarity implies that the required cognitive resources or response needed for executing the task may not be available in the learner's memory. A more overt response could be required for optimal learning from content with unfamiliar subjects. Hulme et al found that memory spans for unfamiliar words are lower than familiar words, which could significantly affect cognitive states. In terms of external stimuli, only timer display provides significant effects on $S P$ and $M$. This could be due to although the same amount of time constraint was allocated to typing task, the estimation of time generated by each person might be different, based on different individual perception of the work amount. Davidson et al [23] argued that typing speed will increase if the individual is able to allow preparation and optimization of typing movement by seeing the text far ahead. The habitual typing behaviour could be broken when stimuli such as time pressure are induced, which could increase their typing speed, but also often leads to mistakes. Davidson's claims could be observed from Table III, when timing and clock are both correlated to decision $D$, which was computed based on the time duration and errors made. But this does not mean that time pressure and clock display could generate strong impact on learner's stress perception and motivation, as much as timer can do.

\section{B. The Correlations of Typing Demand and External Stimuli to User's Stress Perception and Motivation}

Typing demand (Question) gives significant impacts on users' stress perception and motivation. As expected, question with longer length and/or low (language) familiarity increase stress perception and decrease motivation. In terms of external stimuli, only timer display provides significant effects on $S P$ and $M$, but not time pressure and clock display.

The Pearson correlation coefficient tests suggest a few important discoveries to confirm the MADB model. We found some consistent results with what we have found in the menu search task [18]. First, behaviour $B$ is correlated to stress perception $S P$ and motivation $M$. As behaviour produces the outcome (action) of the task, this verifies that the outcome affects the motivation and stress perception in the model. Greater value of behaviour results in lower stress perception but higher motivation. Stress perception is negatively correlated to motivation. When stress perception is higher, motivation becomes lower. Motivation and rational motivation are related to decision, suggests that the motivational state may affect the decision of a student to continue the task. We also observe significant effects of behaviour $B$ on mouse behaviour $B(M)$ and keystroke behaviour $B(K)$ that could be caused by 
the motivation and decision of a student. The significance level of $B$ affecting $B(K)$ is greater than $B(M)$ in the typing task.

Despite consistent results are found, we have also obtained a discrepancy between menu search and typing tasks. First, the correlation between motivation and attitude is not found in the menu search task, but we found significant effect of motivation on attitude in typing task. The reason is typing task considers the attempt to wait until the time is up in the computation of attitude $A$, but on the other side menu search task considers the attempt to revisit a question when calculating $A$. As conclusion, the motivational state of the student is correlated to the attention he or she pays during the typing task, i.e. attempt to wait until the time is up, rather than the attempt to revisit a question as tested in the menu search task.

\section{The Correlations of Behaviour to Mouse Behaviour and Keystroke Behaviour}

Behaviour $B$ provides significant effects on both Mouse Behaviour $B(M)$ and Keystroke Behaviour $B(K)$, but the strength of the effect is stronger on $B(K)$ than $B(M)$ in the typing task, which is expected as typing task involves lesser mouse activities. Significant correlations among behaviour $B$, mouse behaviour $B(M)$ and keystroke behaviour $B(K)$ are found, including mouse click. This shows a great potential of recruiting mouse dynamics and keystroke dynamics analyses in developing an automated cognitive and affective states sensing in e-learning users. Although the correlations of $B$ to $B(M)$ and $B(K)$ also exist in the previous menu search task, however the effect is different. For a greater behaviour value, instead of leading to a slower mouse movements (such as lower mouse speed, higher mouse idle duration and lesser idle occurrences) as found in the menu search task, the mouse movements become faster in typing task. This difference is due to menu search task has a different approach in the experiment as compared to the typing tasks. There is no control or experimental groups in the menu search task as no time constraint is given to the participants. Therefore in the menu search task, $A$ is computed based on the attempt to revisit the question. Since there is no time constraint, the participants' behaviours are not affected by any timing factor. On the other side, $A$ is determined by the passive attempt to wait until the time is up ( $A$ is low if passive attempt occurs) in the typing task. For typing tasks, $B$ improved if the students take proactive step to submit the question earlier. Improvement of $B$ leads to faster mouse movements, as the students would like to submit the answer as fast as possible before the time is up. It is also interesting to observe that mouse speed does not play an important role in this typing task. It is not correlated to any other mouse or keystroke features (although it is correlated to $B)$. We anticipated that this could happen as this task focuses on typing, but surprisingly correlations between other mouse and keystroke features could be observed. This again shows the importance of unifying both mouse and keystroke dynamics to collect user's states so that they complement each other.

\section{The Validation of MADB Model}

We tested the Motivation/Attitude-driven Behaviour (MADB) model applied in the e-learning context and we found major consistencies between menu search and typing

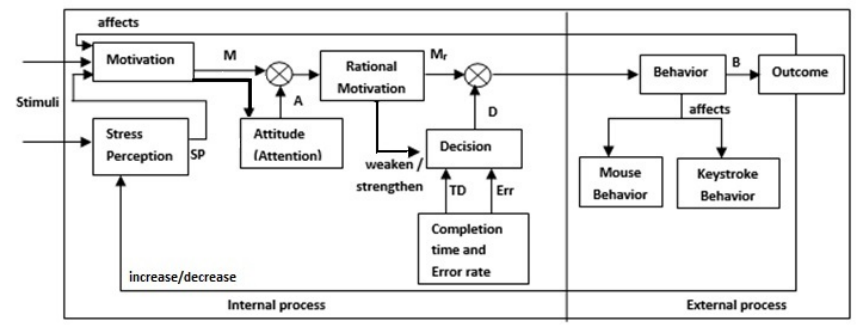

Fig. 2. The Revised MADB Model in the E-Learning Context with Mouse and Keystroke Behaviours

tasks so far. The results corroborates the three hypotheses we made earlier, i.e. (1) typing demand and external stimuli have significant effects on stress perception and motivation; (2) the correlations between typing demand, external stimuli, stress perception and cognitive states are significant; and (3) the correlations of behaviour to mouse behaviour and keystroke behaviour are significant. Based on the results, the revised MADB model in e-learning context, particularly during typing task is found consistent with the proposed MADB model in Section IV-A. The proposed model for typing task is shown in Figure 2. The model is found generally consistent with the model proposed in search task.

\section{CONCLUSION}

Based on the findings from this research, the revised version of MADB model that is applied in the menu search task is found generally consistent with the typing task, although some minor discrepancies are found. Since the impacts of student's behaviour on mouse dynamics and keystroke dynamics could be observed, we strongly believe that there is a potential to compute student's cognitive processes with emotions, motivations and attitude, by observing the changes of mouse behaviour and keystroke behaviour. Therefore a stress measurement model based on mouse and keystroke dynamics can be built. The design and validation of the stress measurement model will be done in our future research.

Our research contains a few limitations. First, our participants come from narrow range of ages and disciplines, and this is not enough for us to generalize our findings. Secondly, homogeneous variance among search tasks cannot be assumed. Each individual has different way to type, and to use mouse and keyboard devices. In addition, lengthy text for Question 5 and Question 6 could lead to aversive feeling, and hence making them reluctantly continuing the task properly, which affected the results.

\section{REFERENCES}

[1] Y. Wang, "On the cognitive processes of human perception with emotions, motivations, and attitudes," International Journal of Cognitive Informatics and Natural Intelligence, vol. 1, no. 4, pp. 1-13, 2007.

[2] M. Sharples, A. Adams, N. Alozie, R. Ferguson, E. FitzGerald, M. Gaved, P. McAndrew, B. Means, J. Remold, B. Rienties, J. Roschelle, K. Vogt, D. Whitelock, and L. Yarnall, "Innovating pedagogy 2015: Open university innovation report 4," The Open University, Milton Keyne, Tech. Rep., 2015.

[3] Y. Rogers, H. Sharp, and J. Preece, Interaction Design: Beyond HumanComputer Interaction, 2nd ed. West Sussex: John Wiley \& Sons Ltd, 2007. 
[4] S. Beilock and G. Ramírez, On the interplay of emotion and cognitive control: Implications for enhancing academic achievement. Elsevier Inc, 2011, vol. 55, ch. 5, pp. 137-169. [Online]. Available: http://dx.doi.org/10.1016/B978-0-12-387691-1.00005-3

[5] G. Hajcak, J. Dunning, and D. Foti, "Neural response to emotional pictures is unaffected by concurrent task difficulty: an event-related potential study," Behavioral Neuroscience, vol. 121, no. 6, p. 1156, 2007.

[6] A. Landowska, "Affective computing and affective learning-methods, tools and prospects," Stara strona magazynu EduAkcja., vol. 1, no. 5, pp. 16-31, 2013.

[7] R. W. Picard, S. Papert, W. Bender, B. Blumberg, C. Breazeal, D. Cavallo, T. Machover, M. Resnick, D. Roy, and C. Strohecker, "Affective learning-a manifesto,” BT Technol. J., vol. 22, no. 4, pp. 253-269, 2004.

[8] J. Hernandez, P. Paredes, A. Roseway, and M. Czerwinski, "Under pressure: sensing stress of computer users," in Proceedings of the 32 d annual ACM conference on Human factors in computing systems. ACM, 2014, pp. 51-60.

[9] A. Ismail, S.-S. Yeo, M. N. Ajis, and N. F. Dollah, "Relationship between occupational stress, emotional intelligence and job performance: An empirical study in malaysia," Theoretical and Applied Economics, no. 10, pp. 3-16, 2009.

[10] M. Heiden, E. Lyskov, M. Djupsjöbacka, F. Hellström, and A. G. Crenshaw, "Effects of time pressure and precision demands during computer mouse work on muscle oxygenation and position sense," European Journal of Applied Physiology, vol. 94, no. 1, pp. 97-106, 2005 .

[11] L. M. Vizer, L. Zhou, and A. Sears, "Automated stress detection using keystroke and linguistic features: An exploratory study," International Journal of Human-Computer Studies, vol. 67, no. 10, pp. 870-886, 2009.

[12] P. Khanna and M. Sasikumar, "Recognising emotions from keyboard stroke pattern," International Journal of Computer Applications, vol. 11, no. 9, pp. 8975-8887, 2010.

[13] G. Tsoulouhas, D. Georgiou, and A. Karakos, "Detection of Learner's Affective State Based on Mouse Movements," Journal of Computing, vol. 3, no. 11, pp. 9-18, 2011. [Online]. Available: https://www.scribd.com/document/75303372/ Detection-of-Learner-s-Affective-State-Based-on-Mouse-Movements

[14] W. Maehr, eMotion: Estimation of User's Emotional State by Mouse Motions. VDM Verlag, 2008.

[15] Y. Lim, A. Ayesh, and M. Stacey, "The effects of menu design on users' emotions, search performance and mouse behaviour," in IEEE 13th Int'l Conf. on Cognitive Informatics \& Cognitive Computing (ICCI*CC'14). IEEE, 2014, pp. 541-549.

[16] _ _ "Using mouse and keyboard dynamics to detect cognitive stress during mental arithmetic," in Intelligent Systems in Science and Information 2014, ser. Studies in Computational Intelligence, K. Arai, S. Kapoor, and R. Bhatia, Eds. Switzerland: Springer, 2015, vol. 591, ch. 21 , pp. 335-350.

[17] _ - "Detecting emotional stress during typing task with time pressure," in Science and Information Conference 2014. London: IEEE Xplore, 2014, pp. 329-338.

[18] _ "The Motivation/Attitude-driven Behavior (MADB) model in ELearning and the Effects on Mouse Dynamics," International Journal of Cognitive Informatics and Natural Intelligence (IJCINI), vol. 10, no. 3, pp. 38-53, 2016.

[19] R. B. Darlington, Regression and Linear Models. McGraw-Hill, 1990.

[20] J. L. Szalma, J. S. Warm, G. Matthews, W. N. Dember, E. M. Weiler, A. Meier, and F. T. Eggemeier, "Effects of sensory modality and task duration on performance, workload, and stress in sustained attention," Hum. Factors J. Hum. Factors Ergon. Soc., vol. 46, no. 2, pp. 219-233, 2004.

[21] S. Tobias and T. Abramson, "Interaction among anxiety, stress, response mode, and familiarity of subject matter on achievement from programmed instruction." Journal of educational psychology, vol. 62, no. 4 , p. 357,1971

[22] C. Hulme, S. Maughan, and G. D. Brown, "Memory for familiar and unfamiliar words: Evidence for a long-term memory contribution to short-term memory span," Journal of Memory and Language, vol. 30, no. 6, pp. 685-701, 1991.

[23] J. E. Davidson and R. J. Sternberg, The psychology of problem solving Cambridge university press, 2003. 